\title{
Hemispheric differences for global/local processing in divided attention tasks: Further evidence for the integration theory
}

\author{
Ronald HÜbner, Gregor Volberg, ANd Tobias Studer \\ Universität Konstanz, Konstanz, Germany
}

\begin{abstract}
Functional hemispheric asymmetries with respect to global/local processing have been observed more reliably in divided-attention than in selective-attention tasks. This difference has been accounted for by assuming that the hemispheres operate differently in the two tasks. In our study, the alternative hypothesis was tested that the interference between the global and local levels is increased under divided attention, and that this makes it necessary to base response selection on a more elaborated mental stimulus representation in which the levels and their content are integrated. Because the hemispheres systematically differ in this integration process (Hübner \& Volberg, 2005), the increased interference between the levels explains why the corresponding asymmetries occur more reliably under divided attention. Two experiments supporting this hypothesis are reported, one with a divided and one with a selective attention task.
\end{abstract}

Over the last few decades, some evidence has been collected for the hypothesis that the local and global levels of hierarchically structured visual stimuli (for examples, see Figure 1) are more efficiently processed within the left and right cerebral hemispheres (LH and RH), respectively. Corresponding effects have been examined in lesion (e.g., Delis, Robertson, \& Efron, 1986; Robertson \& Delis, 1986), electrophysiological (e.g., Evans, Shedden, Hevenor, \& Hahn, 2000; Malinowski, Hübner, Keil, \& Gruber, 2002; Volberg \& Hübner, 2004), response time (e.g., Hübner, 1997; Martin, 1979; Van Kleeck, 1989), and imaging (e.g., Fink et al., 1996; Heinze, Hinrichs, Scholz, Burchert, \& Mangun, 1998) studies. In response time studies, for instance, hierarchical stimuli are projected to the LH or RH by presenting them in the right or left visual field (RVF/LVF), respectively. A speeded response is then required to one level or the other. If the proposed hemispheric differences are valid, level-specific visual field effects (VFEs) would be expected, and indeed, such effects have been observed in several studies (e.g., Hübner, 1997; Martin, 1979).

However, there are also studies in which no VFEs were found. In fact, a recent meta-analysis of response time studies revealed that studies with negative results outnumber those with positive findings (Yovel, Levy, \& Yovel, 2001). Negative results have also occurred in imaging and event-related brain potential (ERP) studies (for an overview, see Volberg \& Hübner, 2004) and in studies with patients (Polster \& Rapcsak, 1994; Schatz, Craft, Koby, \& DeBaun, 2004).

The great variability of results in this area suggests that hemispheric differences for global versus local processing are not a general phenomenon. Rather, it seems that corresponding effects occur only under certain conditions. Thus, an important issue in this field concerns the question of the factors that are responsible for the modulation of hemispheric differences. During the last few years, Hübner and his coworkers (e.g., Hübner, 1997, 1998; Hübner \& Malinowski, 2002; Volberg \& Hübner, 2004, 2006) have shown that response conflict is such a factor (see also Van Kleeck, 1989). In a series of studies, they applied a selective-attention task, in which subjects had to indicate the letter at a prespecified level and ignore the letter at the other level. It turned out that VFEs occurred more reliably for incongruent than for congruent stimuli-that is,

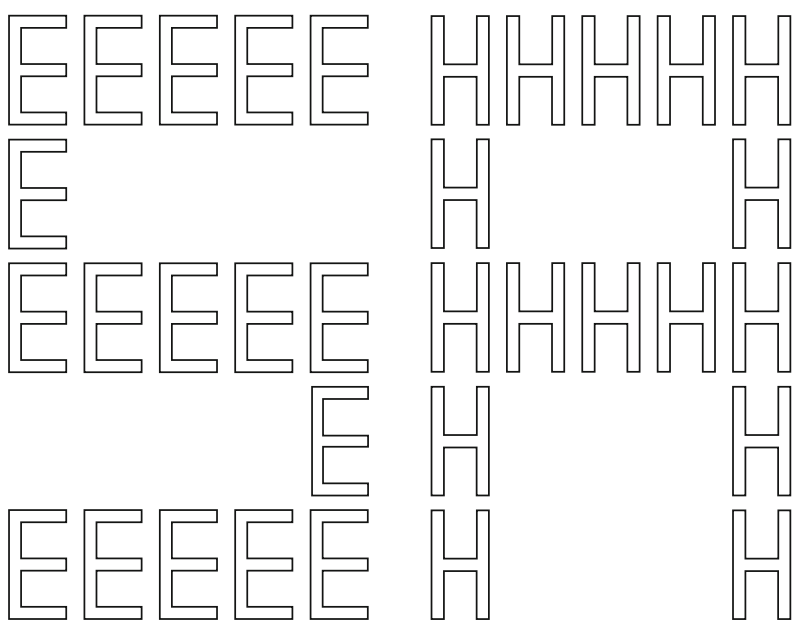

Figure 1. Examples of the hierarchical letters used as stimuli in the present study.

R. Hübner, ronald.huebner@uni-konstanz.de 
for stimuli whose letter at the nontarget level activated a response different from the one at the target level, rather than the same response.

But how can a response conflict affect perceptual hemispheric differences? To provide an account of this phenomenon, Hübner and his coworkers (Hübner \& Malinowski, 2002; Hübner \& Volberg, 2005) proposed the integration theory of global/local processing, which states that hemispheric asymmetries depend on the stimulus representation involved in response selection. According to this theory, it is assumed that there are at least two types of representation. At an early stage of processing, letters are represented without reference to the level at which they appeared. Obviously, if both letters of a hierarchical stimulus activate the same response, as is the case for congruent stimuli, such an early representation is sufficient for selecting the correct response. However, if the two letters activate different responses, a response conflict occurs, which can only be resolved by binding the letters to their level (i.e., by constructing an elaborated representation in which each letter is linked to its level). A crucial assumption of the integration theory is that the two hemispheres do not differ in their capacity for letter identification - that is, with respect to the early stimulus representation - but only with respect to their integration capacity. That is, the RH and LH specialize in binding global and local information, respectively, to the levels in the stimulus. Taken together, these assumptions explain why VFEs occur more reliably for incongruent than for congruent stimuli.

However, there are also conditions in which VFEs occur even though, at first sight, response conflicts seem not to be involved. Such a case is the divided-attention task, in which subjects are instructed to identify which one of two target forms is present in the stimulus, irrespective of the level at which it occurs. The letter at the other level is an irrelevant distractor form that is not mapped to any response. If one considers the corresponding results, it is evident that VFEs occur more reliably under these conditions than under selective attention. For instance, VFEs have been present in almost all response time experiments that required a stimulus identification under divided attention (Brown \& Kosslyn, 1995; Yovel et al., 2001, Experiment 1C).

The divided-attention task has also been successfully applied in imaging studies. For example, Fink et al. (1996) conducted two experiments, in which either selective attention (Experiment 1) or divided attention (Experiment 2) was required. Hemispheric asymmetries occurred in the latter condition only. Similar results were found in an ERP study by Heinze et al. (1998). Whereas under divided attention, local and global processing enlarged the $\mathrm{N} 2$ (negativity at 260-360 msec) amplitude over the left and right hemispheres, respectively, there was no difference between these types of processing under selective attention. Even in lesion studies, VFEs have occurred in divided-attention studies yet been absent under selective attention (Polster \& Rapcsak, 1994), or alternatively, VFEs could be found only under certain conditions (Doricchi \& Incoccia, 1998). Thus, the task seems to play an important role in the manifestation of hemispheric differences.
These results raise the question of why VFEs show up more often under divided than under selective attention. Two studies have addressed this question explicitly. One was a response time study by Yovel et al. (2001), the other an ERP study by Heinze et al. (1998). Yovel et al. concluded from their results that under selective attention, both hemispheres attend to the prespecified level, which minimizes the performance differences between the hemispheres. In contrast, in a divided-attention task, each hemisphere attends to its "preferred" level at the beginning of a trial, because the level at which the target will appear remains unknown until stimulus presentation. Consequently, if the stimulus is projected to one hemisphere and the target appears at its nonpreferred level, attention has to be redirected, which delays processing. This explains why VFEs are enhanced under divided attention in comparison with selective attention.

Alternatively, Heinze et al. (1998) assumed that in the selective-attention task subjects focus an attentional zoom lens (Eriksen \& St. James, 1986) on the prespecified level, which allows them to process only the relevant range of spatial frequencies. In contrast, when attention has to be divided between levels, subjects cannot select a specific spatial frequency range in advance and, therefore, must process the complete range contained in the stimulus. Under the further assumption that the LH and the RH specialize in the processing of relatively high and low spatial frequencies, respectively (Ivry \& Robertson, 1998), processing would be distributed between hemispheres only under divided attention, which accounts for the hemispheric differences.

Although the two proposed mechanisms (Heinze et al., 1998; Yovel et al., 2001) can explain why hemispheric differences are more pronounced under divided than under selective attention, these mechanisms are rather specific and, therefore, have difficulties accounting for the effects of other important factors. In particular, they cannot explain why response conflict supports the appearance of VFEs in selective-attention tasks (Hübner \& Malinowski, 2002; Hübner \& Volberg, 2005; Van Kleeck, 1989; Volberg \& Hübner, 2004, 2006). Therefore, it would be desirable to have a more general theory. Fortunately, such a theory is already available, in the integration theory of global and local processing (Hübner \& Volberg, 2005). In the present study, we show that this theory can explain not only why response conflicts modulate VFEs, but also why VFEs occur more reliably under divided attention.

If, as assumed by the integration theory, the hemispheres differ only in their capacity for binding the levels with their contents, it then follows that such a binding must always have taken place under divided attention. However, why should subjects always use an integrated representation of the letters and levels, even though the level of the target is obviously irrelevant for the response? A possible reason could be that the distractors, although completely irrelevant, nevertheless activate the alternative response to such an extent that response selection cannot be based on a pure letter representation. Such a response activation is not implausible, since distractors are often rather similar to the potential targets. Indeed, similarity relations between 
distractors and targets have even been used to define congruency relations for the divided-attention task.

Lamb and Robertson (1989), for instance, used $H$ and $S$ as target letters, which were combined with the distractors $A$ and $E$. If all letters are constructed only of horizontal and vertical lines (see Figure 2), and if similarity is defined by the overlap of segments, then the distractor $A$ is more similar to the target $H$ than to the target $S$, whereas the distractor $E$ is more similar to $S$ than to $H$. Thus, the distractors $A$ and $E$ should activate the responses associated with the targets $H$ and $S$, respectively. As a consequence, if the target $H$ is combined with the distractor $E$, or the target $S$ with the distractor $A$, there should be a response conflict. Therefore, these combinations were considered incongruent, whereas the remaining combinations were considered congruent. Indeed, congruency defined in this way produced the expected effects (see, e.g., Heinze et al., 1998; Lamb \& Robertson, 1989).

From the viewpoint of the integration theory, the fact that VFEs have reliably been observed in divided-attention tasks, irrespective of congruency relations, suggests that supposedly congruent stimuli are actually incongruent. This assumption is also not implausible. For instance, if we examine a hierarchical stimulus with the target letter $H$ and distractor $A$, which would be considered congruent (see, e.g., Lamb \& Robertson, 1989), it is obvious that $A$ shares visual features not only with the actual target $H$, but also with the alternate target $S$, even if to a smaller extent (see Figure 2). However, even a small feature overlap might activate the alternative response to a substantial extent.

If both responses are activated, the subject cannot be sure which target letter is actually present in the current stimulus. How can this problem be solved? We supposed that subjects would proceed in the same way as in the case of a response conflict under selective attention, by basing their response on a more complete stimulus representation. Although in a divided-attention task it would, in principle, be sufficient merely to improve the representation of the identity of the target letter in the current stimulus, it is likely that during this process the level is also bound to the identity. Because the hemispheres differ in this binding process, this could explain why VFEs show up more reliably under divided attention. Whether these processes take place for incongruent as well as for congruent stimuli was a question we tested in our first experiment.

\section{EXPERIMENT 1}

In this experiment, a divided-attention task was used and congruency was varied by the degree of similarity between target and distractor. However, in addition to the usual congruent and incongruent distractors, there was also a "neutral" letter $O$, which we hoped would be more dissimilar to the targets than their respective congruent distractors. If we simply count the number of overlapping elements, $O$ does not differ more from the targets than the other distractors. However, we assumed that similarity is determined not only by the number of overlapping segments, but also by other, more qualitative features. For instance, it was thought that the missing central bar and

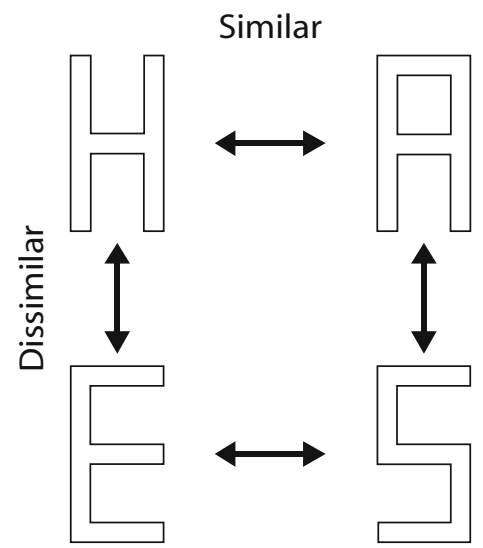

Figure 2. Similarity relations between the targets $(H$ and $S)$ and distractors $(E$ and $A)$. Similarity is defined as the number of corresponding contours. The letters $H$ and $A$ differ only in the upper horizontal bar, and the letters $E$ and $S$ only in the position of the lower vertical bar. In contrast, the letters $H$ and $E$, on the one hand, and $A$ and $S$, on the other, differ by four and three bars, respectively.

closed form were characteristic features of the letter $O$, so that it could easily be differentiated from the other letters. Consequently, the $O$ should activate the target representations to a lesser extent than do the other distractors.

Our aim was to demonstrate that the usual "congruent" distractors also produce some response conflict relative to the neutral distractor. Furthermore, if this is the case, then according to the integration theory, VFEs should arise for the congruent stimuli, as would be usual in divided-attention tasks. However, for neutral stimuli, VFEs should be absent, or at least smaller than those for congruent stimuli.

Exactly what data pattern do we expect if the proposed hemispheric differences are present? For responses to targets at the global level, the mean response time for RVF stimuli, $\mathrm{RT}_{\mathrm{G}}(\mathrm{RVF})$, should be longer than the mean response time for $\mathrm{LVF}$ stimuli, $\mathrm{RT}_{\mathrm{G}}(\mathrm{LVF})$. In other words, the visual field effect for the global level, defined by $\mathrm{VFE}_{\mathrm{G}}=$ $\left[\mathrm{RT}_{\mathrm{G}}(\mathrm{RVF})-\mathrm{RT}_{\mathrm{G}}(\mathrm{LVF})\right]$, should be greater than zero. In contrast, for responses to targets at the local level, the opposite result should hold - that is, $\mathrm{VFE}_{\mathrm{L}}=\left[\mathrm{RT}_{\mathrm{L}}(\mathrm{LVF})-\right.$ $\left.\mathrm{RT}_{\mathrm{L}}(\mathrm{RVF})\right]>0$. Because a main effect of VF can occur for various reasons, it is important that the contrasting levelspecific VFEs be analyzed together. This is usually done by means of an ANOVA. If both level-specific VFEs are in the expected direction, the analysis should reveal a significant interaction between the target level and VF.

However, an ANOVA is not a very efficient means of testing the predicted specific pattern of VFEs, because it tests for all possible types of interaction between target level and VF. Therefore, one gains statistical power by directly testing the proposed specific interaction between target level and VF by means of a one-sided $t$ test, as has been done, for instance, by Yovel et al. (2001). This is equivalent to the test of whether the combined VFE is significant - that is, whether $\mathrm{VFE}_{\mathrm{G}}+\mathrm{VFE}_{\mathrm{L}}>0$. Given a significant combined VFE, we could also test the individual VFEs, so that three outcomes would be possible: 
Both, one, or none of the VFEs could be significant. To differentiate between these results, we will denote the case in which both level-specific VFEs are significant as a strong combined VFE, and the other two cases as weak combined VFEs.

In the present study, we used these specific tests, in addition to an overall ANOVA, to analyze response times and error rates in the three congruency conditions. It was predicted that congruent and incongruent stimuli would produce significant VFEs. With respect to neutral stimuli, the question was open whether they would also produce VFEs, but the result should depend on the induced response conflict. That is, if they produce a small or no response conflict (i.e., if the corresponding response time is substantially faster than that for congruent stimuli), then VFEs with the neutral stimulus should be absent or at least significantly smaller than those with the congruent stimuli.

\section{Method \\ Subjects. Sixteen students of the Universität Konstanz participated in the experiment ( 8 female, 8 male; mean age, 23.4 years). They re- ceived either course credit or a fee of $€ 5 / \mathrm{h}$. All subjects were right- handed by self-report and had normal or corrected-to-normal vision. \\ Apparatus. The stimuli were shown on a 21 -in. color monitor with a vertical refresh rate of $75 \mathrm{~Hz}$ at a resolution of $1,280 \times 1,024$ pixels. A two-button mouse served as the response device. Stimulus presentation and response registration were controlled by the same personal computer.}

Stimuli. The stimuli were hierarchical letters, which were constructed from local letters in a $5 \times 5$ grid (see Figure 1 ). The letters $H$ and $S$ served as targets. They were combined with the letter $A, E$, or $O$, resulting in 12 different compound stimuli. If we denote the hierarchical stimuli by letter pairs such as $H A$, with the first letter in a pair denoting the global letter and the second the local letter, then $H A, S E, A H$, and $E S$ were considered as congruent; $H E, S A$, $E H$, and $A S$ as incongruent; and $H O, S O, O H$, and $O S$ as neutral. The size of the local letters was $0.6^{\circ}$ horizontally and $0.7^{\circ}$ vertically, whereas that of the global letter was $3.3^{\circ} \times 4.5^{\circ}$ of visual angle. The letter elements of the stimulus were drawn as white outlines on a black background, with the inner edge of the stimulus aligned with the screen midline. Accordingly, the eccentricity was $1.65^{\circ}$ of visual angle, measured from fixation to the midpoint of the stimulus. In the vertical dimension, the stimuli were centered.

Procedure. The subjects were seated in front of a monitor at a viewing distance of $110 \mathrm{~cm}$. A chinrest prevented head movements during the experiment. Each trial began with a 300-msec presentation of a fixation cross in the center of the screen. After a blank screen of $300 \mathrm{msec}$, a hierarchical letter was presented to the LVF or to the RVF for $93 \mathrm{msec}$. The task was to indicate as quickly and accurately as possible which of the predefined target letters $(H$ or $S)$ was present in the stimulus. The subjects responded with the index or middle finger of the same hand. Half of the subjects pressed a left key for the letter $H$ and a right key for the letter $S$, and the other half had the opposite letter-to-key assignments. In addition, the response hand (left or right) was balanced across subjects. Wrong responses were signaled by a tone. After each response, there was an interval of $1,000 \mathrm{msec}$ before the next trial started.

Three factors were varied: target level (global or local), VF (LVF or RVF), and congruency (congruent, incongruent, or neutral). All factors were randomized. The subjects performed 12 blocks of 64 trials each. Accordingly, each condition was covered by 96 observations per subject.

\section{Results}

Both the response times for trials with correct responses and the error rates were first subjected to a three-factorial
ANOVA with repeated measures on the involved factors. All significant results are reported. Furthermore, the data were also analyzed by planned individual tests for the expected VFEs.

Response times. The analysis revealed a main effect for congruency $[F(2,30)=20.67, p<.001]$. Planned comparisons showed that the responses to neutral stimuli were significantly faster than those to congruent stimuli [476 vs. $499 \mathrm{msec} ; t(63)=5.10, p<.001$ ], which in turn were significantly faster than those to incongruent stimuli [524 msec; $t(63)=3.31, p<.01]$. Furthermore, the two-way interaction between congruency and target level was reliable $[F(2,30)=15.69, p<.001]$. This interaction was due to the fact that the congruency effect (i.e., the response time difference between congruent and incongruent stimuli) was much larger for local targets than for global ones ( 48 vs. $4 \mathrm{msec}$ ). On the other hand, the difference between neutral and incongruent stimuli was similar for the two levels ( $49 \mathrm{vs} .48 \mathrm{msec}$ ).

There was also a reliable two-way interaction between target level and VF $[F(1,15)=20.67, p<.001]$; however, this interaction was qualified by a further, threeway interaction between target level, VF, and congruency $[F(2,30)=4.32, p<.05]$. Because the VFEs were in the expected direction in the three congruency conditions (as can be seen in Figure 3), this interaction must have been due to different effect sizes. This was confirmed by individual tests. For the incongruent condition, not only was the combined VFE significant $[t(15)=3.02$, $p<.001]$, so were the individual VFEs for the global $[14 \mathrm{msec} ; t(15)=2.53, p<.05]$ and local $[19 \mathrm{msec}$; $t(15)=2.64, p<.01]$ levels. The same pattern resulted for the congruent condition: The combined VFE was significant $[t(15)=4.64, p<.001]$, as were the individual VFEs for the global [19 msec; $t(15)=3.28, p<.01$ ] and local $[15 \mathrm{msec} ; t(15)=2.67, p<.01]$ levels. For the neutral condition, the combined VFE was also significant $[t(15)=2.49, p<.05]$. However, of the individual VFEs in this condition, only the one for the local level $(8 \mathrm{msec})$ was significant $[t(15)=2.94, p<.01]$, not the one for the global level (2 msec). Moreover, a further test comparing the combined VFEs, revealed that they were significantly smaller for the neutral stimuli than for the congruent ones $[t(15)=3.17, p<.01]$.

Error rates. The ANOVA on the arcsine-transformed error rates revealed a main effect for the factor target level $[F(1,15)=7.78, p<.05]$. This effect shows that subjects produced fewer errors in the global $(2.10 \%)$ than in the local $(3.03 \%)$ condition. Also the main effect of congruency was reliable $[F(2,30)=12.7, p<.001]$. Responses to congruent $(1.92 \%)$ and neutral $(2.07 \%)$ stimuli were less erroneous than those to incongruent stimuli (3.71\%). No VFEs were significant, which shows that there was no speed-accuracy trade-off with these effects.

\section{Discussion}

In this experiment, we investigated why VFEs occur more reliably in divided-attention than in selectiveattention tasks, irrespective of the congruency of the stimuli. We hypothesized that this occurs because of the 
relatively large degree of similarity between the targets and distractors in the former task. More specifically, we hypothesized that stimuli that are typically considered to be congruent also produce some response conflict, and that this conflict in turn leads to VFEs (Hübner \& Volberg, 2005). Therefore, we also included stimuli with neutral distractors, which were thought to be even less similar to the targets than the "congruent" distractors.

As have other researchers (e.g., Lamb, Robertson, \& Knight, 1989), we found the usual congruency effects (i.e., congruent distractors led to faster responses than incongruent ones). However, the fastest responses occurred for the neutral distractor. This demonstrates that even congruent distractors produced some amount of response conflict, so the term congruent can only be understood in a relative sense under these conditions. In any case, the congruency effects for the different stimulus types were as expected.

The crucial question was whether the different congruency conditions modulated the VFEs in the expected way. Indeed, as predicted by the integration theory, a strong combined VFE occurred only for stimuli that produced a substantial response conflict, as was the case for congruent and incongruent stimuli. In contrast, neutral stimuli merely produced a weak combined VFE (see Figure 3). Moreover, this VFE was significantly smaller than that for congruent stimuli. Although this data pattern holds only for the response times, the error rates do not indicate any speed-accuracy trade-off.

Thus, taken together, the results of the present experiment confirm the predictions derived from the integration theory of global/local processing. It seems that the similarity between target and distractor for incongruent as well as for congruent stimuli made it difficult to select the correct response using letter information alone. Even the congruent distractor activated the opposite response to a substantial degree. Consequently, in order to solve this ambiguity, the subjects had to construct a more elaborated target representation, which automatically also encompassed level information. Because we assume that the hemispheres differ in their capacity for binding the letters to their levels, this more elaborate representation explains why strong VFEs occurred in these conditions. Neutral distractors, on the other hand, activated the alternative response to a relatively small degree. Therefore, with those distractors, response selection could often occur directly, through letter information alone.

Obviously, the alternative accounts proposed by Heinze et al. (1998) and Yovel et al. (2001) cannot explain the present data. According to their view, hemispheric asymmetries should generally be strong in divided-attention conditions and not depend on response conflicts.

\section{EXPERIMENT 2}

The results of our first experiment show that VFEs occur in divided-attention tasks for the same reason that they occur for incongruent stimuli in selective-attention tasks. Thus, the underlying mechanisms do not differ qualitatively between the two tasks. However, it can be assumed that the tasks do differ with respect to the use of early filter mechanisms that allow the subjects to select the response by letter information alone (see Hübner \& Volberg, 2005). Clearly, if the target level is known in advance, as in the selective-attention task, subjects can focus their attention on that level and suppress irrelevant information from the other level. If filtering were perfect, there would be no need to integrate the letter and level information.

Usually, however, early filtering is imperfect, so that information from the irrelevant level may affect the response selection process. The efficiency of the filtering process depends on various factors. For instance, it has been shown that, if the target level is randomized across trials in a selective-attention task, the efficiency is reduced (Hübner, 1997; Hübner \& Malinowski, 2002). An extreme situation in this respect seems to occur when attention has to be divided between levels. In this case, no early filtering is possible, and even weak conflicting signals make it necessary to rely on elaborated target representations. This is the reason why an irrelevant letter, even if its similarity to the alternative target is relatively small, is sufficient to produce VFEs.

It follows from these considerations that, if congruency is defined in the same way as under divided attention, the VFEs should generally be reduced, if not absent, under selective attention. This hypothesis was tested in the next experiment, in which a selective-attention task was used. We expected congruency effects to occur, if at all, only for incongruent stimuli. Accordingly, VFEs should occur only in this condition.

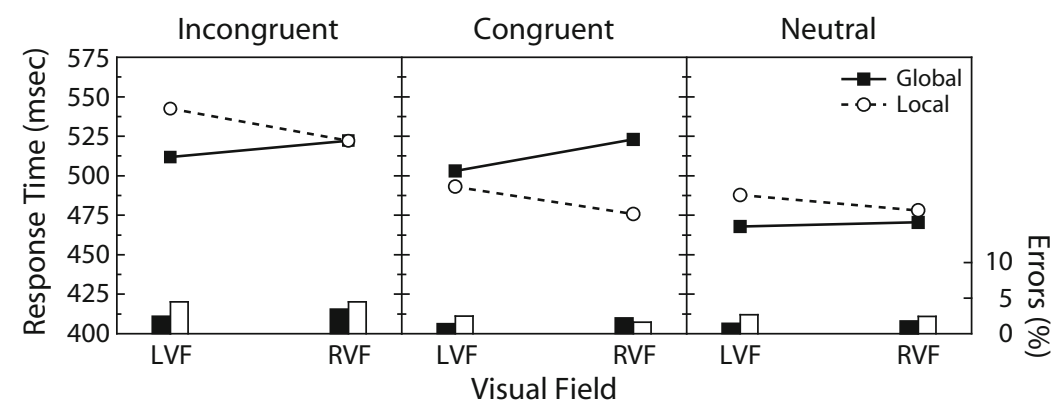

Figure 3. Interaction between target level and visual field for the three stimulus conditions of Experiment 1. 


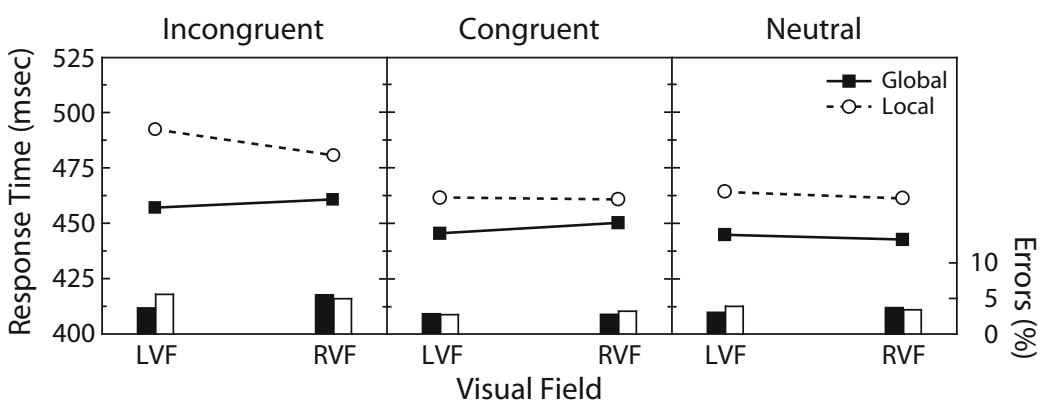

Figure 4. Interaction of target level, visual field, and congruency in Experiment 2.

\section{Method}

Sixteen right-handed students of the Universität Konstanz participated in the experiment ( 8 male, 8 female; mean age, 23.5 years). All of them had normal or corrected-to-normal vision.

The stimuli were identical to those of the first experiment. However, in contrast to Experiment 1, a selective-attention task was used. The trials started with $300-\mathrm{msec}$ presentation of a cue in the center of the screen. The cue letters $L$ and $G$ indicated whether attention had to be directed at the local or the global level, respectively. After a blank screen of $300 \mathrm{msec}$, the stimuli were presented to the LVF or the RVF. The subjects were told to indicate as quickly and accurately as possible whether the letter $H$ or $S$ was present at the cued level. Half of the subjects responded with a left buttonpress for the letter $H$ and a right buttonpress for the letter $S$; with the other half, this mapping of letters to keys was reversed. As in Experiment 1, the response hand was balanced across subjects and wrong responses were indicated by a warning tone. After a response was made, an interval of $1,000 \mathrm{msec}$ followed before the next trial started.

Three factors were varied: target level (global or local), VF (LVF or RVF), and congruency (congruent, incongruent, or neutral). All of these factors were randomized. After two training blocks, the subjects were given 16 blocks of 72 trials each, so that each of the 12 conditions was covered by 96 observations per subject.

\section{Results}

The response times of correct trials were entered into a three-factorial ANOVA with repeated measures on all factors. All significant results are reported. Moreover, as in Experiment 1, the data were further analyzed by planned specific tests.

Response times. The ANOVA revealed a significant main effect of target level $[F(1,15)=18.83, p<.001]$, indicating that responses to the global level $(450 \mathrm{msec})$ were faster than those to the local level $(470 \mathrm{msec})$. The factor congruency was also reliable $[F(2,30)=14.74, p<$ $.001]$, with responses to incongruent stimuli $(473 \mathrm{msec})$ slower than those to congruent $(455 \mathrm{msec}$ ) and neutral $(453 \mathrm{msec})$ ones. The interaction between these factors was marginally significant $[F(2,30)=2.90, p<.07]$, indicating that the interference from the global to the local level was stronger than vice versa $(26 \mathrm{msec} v \mathrm{vs} .11 \mathrm{msec})$. The three-way interaction between target level, VF, and congruency failed to reach significance, but it did show a trend in the expected direction $[F(2,30)=2.14, p=.14]$. However, as argued in the introduction, an ANOVA is not a very efficient test of our hypotheses. Therefore, we analyzed the data further in a hypothesis-driven way.

The combined and individual VFEs were analyzed in more detail by computing planned tests for each of the three congruency conditions. These analyses revealed a significant combined VFE for the incongruent condition $[t(15)=2.14, p<.05]$. Of the individual VFEs in this condition, the one for the local level $(10 \mathrm{msec})$ just failed to reach significance $[t(15)=1.71, p=.054]$, whereas that for the global level $(3 \mathrm{msec})$ was not reliable. The combined VFEs for the other two congruency conditions were not analyzed, because in each condition the effect for one level was not in the expected direction (with neutral distractors, $6 \mathrm{msec}$ local, $-2 \mathrm{msec}$ global; with congruent distractors, $-3 \mathrm{msec}$ local, $5 \mathrm{msec}$ global). These results are depicted in Figure 4.

Error rates. The analysis of the arcsine-transformed error rates revealed a significant effect of congruency $[F(2,30)=7.40, p<.001]$, indicating that more errors occurred in the incongruent (4.96\%) than in the congruent $(2.91 \%)$ or neutral $(3.57 \%)$ condition. There was also a significant three-way interaction between target level, VF, and congruency $[F(2,30)=4.54, p<.05$; see Figure 4]. The specific tests revealed that a reliable combined VFE occurred only for the incongruent condition $[t(15)=3.66$, $p<.001]$. Of the level-specific VFEs in this condition, that for the global level $(1.92 \%)$ was significant $[t(15)=$ $4.20, p<.001]$, whereas that for the local level $(0.987 \%)$ was not reliable. Thus, there was a weak combined VFE for the incongruent condition.

\section{Discussion}

In this experiment, we tested whether the similarity relations between letters, as defined in Experiment 1, were strong enough to also produce congruency effects under focused attention, and if so, whether this effect would have the predicted consequences for the occurrence of VFEs. As our results show, congruency defined by letter similarity produced reliable effects even with a focused-attention task. On average, responses were about $20 \mathrm{msec}$ faster for congruent and neutral distractors than for the incongruent ones. However, in comparison with the divided-attention task in our first experiment, this effect was only about half the size. Furthermore, in contrast to Experiment 1, there was no difference in response times between neutral and congruent distractors.

These results demonstrate that under focused attention, subjects were generally more successful at filtering out irrelevant information. As a result, the relatively weak activations caused by the "congruent" stimuli did not produce a 
substantial response conflict. Furthermore, in accord with the predictions of our integration theory, there was only a weak combined VFE, and only for incongruent distractors.

\section{GENERAL DISCUSSION}

Our aim in this study was to further investigate the origins of hemispheric differences in the processing of the global and local levels of hierarchical stimuli. The challenge was to explain why the corresponding VFEs occur more reliably with divided-attention than with selectiveattention tasks. Accounts proposed so far for explaining this difference (Heinze et al., 1998; Yovel et al., 2001) are rather restricted and, therefore, cannot explain our additional results. For instance, in various studies from our group (Hübner, 1997, 1998; Hübner \& Malinowski, 2002; Hübner \& Volberg, 2005; Malinowski et al., 2002; Volberg \& Hübner, 2004, 2006), we have demonstrated that VFEs also occur reliably with selective-attention tasks, but only for incongruent stimuli- that is, when global and local information activate competing responses - and mainly when the target level is randomized. This pattern cannot be explained by the current accounts.

Therefore, in this study we investigated whether the integration theory of global/local processing (Hübner \& Volberg, 2005), which was developed to account for the result that VFEs occur most reliably for incongruent stimuli under selective attention, can also explain the success of divided-attention tasks in this respect. The basic assumption of this theory is that at an early stage of processing, letter identity is available separately from information about the level at which the letter occurs. Consequently, under optimal conditions, a target letter can quickly be categorized without reference to level information. However, under less optimal conditions (e.g., when the letters at the two levels activate conflicting responses), letter and level information has to be integrated in order to select the correct response. A crucial assumption of the integration theory with respect to the present objective is that the hemispheres do not differ at the early letter identification stage, but only at the later integration stage. This explains why in selective-attention tasks VFEs occur for incongruent stimuli, but not for congruent or neutral ones.

The question was whether the integration theory can also explain the results observed with divided-attention tasks, particularly the fact that VFEs seemed to occur irrespective of congruency relations. In corresponding studies, congruency was defined by the degree of similarity between the shape of target and distractor letters (see Figure 2; Lamb et al., 1989): If the distractor was more similar in shape to the current target than to the alternative target, it was defined as congruent. In contrast, if the distractor was more similar to the alternative target than to the actual target, it was considered incongruent. The reduced performance for incongruent stimuli relative to congruent ones, as observed in other experiments (e.g., Heinze et al., 1998; Lamb \& Robertson, 1989) as well as in the present ones, confirms that congruency defined in this way has the intended effect.
However, a closer look at the details reveals that differences exist between congruency as defined by the stimulus-response mapping in selective-attention tasks, and congruency as defined by similarity between letters. Although responses to congruent stimuli in dividedattention tasks are faster than those to incongruent stimuli, the congruent stimuli might nevertheless produce some conflict, because they still resemble the alternative target to some degree. Thus, our idea was that, under divided attention, congruent stimuli produce some degree of response conflict that, according to the integration theory, could explain the observed VFEs in this condition.

This hypothesis was tested in our first experiment. For comparison, we also used a neutral distractor, which was thought to be less similar to each target than was that target's congruent distractor. As expected, the subjects responded faster to the neutral than to the congruent stimuli. This confirmed our suspicion that, under divided attention, the "congruent" stimuli nonetheless produced some response conflict. Furthermore, it turned out that, as predicted by the integration theory, strong combined VFEs occurred only for the incongruent and congruent stimuli. Moreover, the combined VFE in the neutral condition was significantly smaller than the VFE in the congruent condition. However, a weak combined VFE occurred even in the former condition, suggesting that even neutral stimuli produced response conflict to some extent.

The results of our first experiment show that the mere fact that subjects have to divide their attention between levels is not responsible for the more reliable VFEs under divided attention, but instead the fact that response conflicts are also present for the so-called congruent stimuli. Such conflicts require subjects to base their response selection on an elaborated target representation in the majority of trials, which then brings hemispheric differences into effect. However, it seemed reasonable to assume that dividing attention between levels should nevertheless play some role for the occurrence of VFEs. We hypothesized that the similarity between letters could produce substantial response conflicts only because the division of attention prevented subjects from applying early filter mechanisms (Hübner \& Volberg, 2005). In contrast, when the target level is known in advance in a selective-attention task, subjects are able to allocate more resources to the relevant level and to filter out irrelevant information from the nontarget level. As a consequence, the weakly processed distractors interfere with performance only if they produce a strong signal. From these considerations, we derived the hypothesis that similarity relations between letters should produce less response conflict in a selectiveattention task, and consequently that VFEs would occur only for incongruent stimuli, if at all.

This hypothesis was tested in Experiment 2, in which a selective-attention task was used. However, unlike former studies of this type, we defined congruency according to the similarity between letters, as in Experiment 1. As expected, congruency effects were generally reduced and were reliable only for incongruent stimuli, with no difference between congruent and neutral stimuli. Accord- 
ingly, as predicted by the integration theory and observed in other studies applying a selective-attention task (e.g., Hübner \& Malinowski, 2002; Volberg \& Hübner, 2004), (weak) VFEs occurred only for incongruent stimuli.

Overall, our results show that divided-attention tasks are favorable for observing VFEs, because they prevent subjects from using early filter mechanisms to reduce interference from the nontarget level. Consequently, in such tasks even a weak disturbing signal from the irrelevant level produces response conflict, requiring the subject to construct an elaborated target representation in order to select the correct response. Presumably, this elaborated representation also led automatically to the integration of the level. Because the hemispheres systematically differ in their integration capacity for the two levels (Hübner \& Volberg, 2005), robust VFEs can be observed under these conditions. Thus, the integration theory not only explains the modulating effect of congruency on VFEs for selective-attention tasks, but also the greater success of divided-attention tasks in obtaining these effects. The alternative accounts proposed by Heinze et al. (1998) and Yovel et al. (2001) cannot explain the present pattern of results, mainly because neither account predicts any differences between congruent, incongruent, and neutral conditions with respect to VFEs.

The integration theory can also explain another phenomenon that has been observed with divided-attention tasks. VFEs in global/local processing have occurred regularly only when two or more forms served as targets, not when only one form needed to be detected (detection task). It is reasonable to assume that in the detection task subjects are also able to use an early filter. However, unlike in a selective-attention task, in which a level filter is applied for suppressing information from the irrelevant level, a form filter (Paquet, 1992; Paquet \& Merikle, 1988 ) is presumably used in the detection task. If only one form has to be detected, a filter can be constructed that is narrowly tuned to that form. Consequently, distractors can activate the alternative response only weakly, allowing the response to be selected at an early stage of stimulus representation. Accordingly, no VFEs should occur under these conditions. This is exactly what has been reported in the literature (Blanca Mena, 1992; Han, Liu, Yund, \& Woods, 2000).

An ongoing controversy related to our research is whether hemispheric differences for global and local processing occur during "early" perceptual processing or arise only at "later" attentional stages. For example, in an imaging study, Heinze et al. (1998; see also Sasaki et al., 2001) found no hemispheric differences between global and local processing in the primary visual cortex, and concluded that hemispheric differences occur only at later stages of processing. In contrast, other authors have found differential activity in the visual cortex if the task was more demanding - for instance, if subjects frequently had to switch attention between levels (Fink et al., 1996) or if the information at a given level was degraded (Lux et al., 2004). Likewise, the results of ERP experiments are also inconclusive. In the majority of such studies, hemispheric asymmetries have not occurred before $250 \mathrm{msec}$ after stimulus onset - that is, at a relatively late stage of processing (Heinze et al., 1998; Malinowski et al., 2002; Volberg \& Hübner, 2004). However, differences in the early ERP components have also been reported (Evans et al., 2000). Thus, the question of whether hemispheric differences occur at earlier perceptual or later attentional stages of processing remains unresolved.

Obviously, the present results are more compatible with an attentional explanation. It is important to note that the stimuli used in the congruent conditions of Experiments 1 and 2 were identical. Thus, if VFEs were caused by hemispheric differences in perceptual processing, the same VFEs should have occurred under selective and divided attention. However, this was not the case, which suggests that hemispheric differences in global and local processing are not perceptual. Rather, it seems that hemispheric differences occur only if an elaborated stimulus representation is required for performing a task - in other words, at a later stage.

If one considers only the present results, one might question whether the integration theory is necessary for explaining the relation between response conflict and VFEs. At first glance, it seems sufficient to assume that congruency is favorable for VFEs and that the larger the congruency effect, the larger the VFEs. However, the relation is not that simple. First of all, even if there were such a simple quantitative relationship, it alone would tell us nothing about the specific underlying mechanisms. Moreover, as has already been shown by Hübner and Malinowski (2002), there is no simple quantitative relationship between the amount of response conflict and the size of VFEs. This was also confirmed by the results of our Experiment 1. Even though incongruent stimuli produced stronger response conflict than did congruent stimuli, the corresponding VFEs did not differ. If they did differ at all, the trend was for them to differ in the direction opposite to expectations.

In a related objection, one might suspect that congruency modulates the general speed of processing, which in turn affects VFEs in the observed way. For the present study, this argument can even be extended to the comparison between the two experiments. In Experiment 2, not only were the VFEs smaller than in Experiment 1, the overall latencies were as well. Thus, we face the dilemma that response conflict usually covaries with response (i.e., processing) time. Consequently, the question of which variable is ultimately responsible for the variation of VFEs remains open. To attack this problem, our group performed a global/local study in which a variable mapping between the involved letters and responses was used (Volberg \& Hübner, 2007). As we had hoped, this had the effect of balancing latencies for the congruent and incongruent stimuli. Nevertheless, as predicted by our integration theory, VFEs occurred only for incongruent stimuli. This shows that processing time alone is not the crucial variable.

Furthermore, to show that response conflicts or corresponding latency variations are generally not necessary for inducing the integration between levels and their content, or for inducing the corresponding VFEs, Hübner and Volberg (2005) used an entirely different procedure, in 
which unspeeded responses to masked hierarchical letters were required. Congruency relations were not even defined in these experiments. It turned out that the subjects often confused the letters at the two levels when the stimuli were only present for a short time, showing that letters and levels are unbound at an early stage of processing. Only when the stimuli could be processed for a sufficient amount of time were confusions rare, indicating that subjects had constructed elaborated stimulus representations.

Overall, our results strongly support the integration theory of global and local processing. Thus, this theory turns out to currently be the most parsimonious account for explaining a variety of results and phenomena in the area of hemispheric asymmetries for global versus local processing.

\section{AUTHOR NOTE}

This research was supported by a grant from the Deutsche Forschungsgemeinschaft $(\mathrm{Hu} 432 / 7)$ to the first author. We thank our two anonymous reviewers for their helpful comments. Correspondence relating to this article may be sent to R. Hübner, Universität Konstanz, Fachbereich Psychologie, Fach D29, D-78457 Konstanz, Germany (e-mail: ronald .huebner@uni-konstanz.de).

\section{REFERENCES}

Blanca Mena, M. J. (1992). Can certain stimulus characteristics influence the hemispheric differences in global and local processing? Acta Psychologica, 79, 201-217.

Brown, H. D., \& Kosslyn, S. M. (1995). Hemispheric differences in visual object processing: Structural versus allocation theories. In R. J. Davidson \& K. Hugdahl (Eds.), Brain asymmetry (pp. 77-97). Cambridge, MA: MIT Press.

Delis, D. C., Robertson, L. C., \& Efron, R. (1986). Hemispheric specialization of memory for visual hierarchical stimuli. Neuropsychologia, 24, 205-214.

Doricchi, F., \& INCOCCIA, C. (1998). Seeing only the right half of the forest but cutting down all the trees? Nature, 394, 75-78.

Eriksen, C. W., \& St. James, J. D. (1986). Visual attention within and around the field of focal attention: A zoom lens model. Perception \& Psychophysics, 40, 225-240.

Evans, M. A., Shedden, J. M., Hevenor, S. J., \& Hahn, M. C. (2000). The effect of variability of unattended information on global and local processing: Evidence for lateralization at early stages of processing. Neuropsychologia, 38, 225-239.

Fink, G. R., Halligan, P. W., Marshall, J. C., Frith, C. D., FrackowiaK, R. S. J., \& Dolan, R. J. (1996). Where in the brain does visual attention select the forest and the trees? Nature, 382, 626-628.

HaN, S., Liu, W., Yund, E. W., \& Woods, D. L. (2000). Interactions between spatial attention and global/local feature selection: An ERP study. NeuroReport, 11, 2753-2758.

Heinze, H. J., Hinrichs, H., Scholz, M., Burchert, W., \& Mangun, G. R. (1998). Neural mechanisms of global and local processing: A combined PET and ERP study. Journal of Cognitive Neuroscience, 10, 485-498.

HÜBNER, R. (1997). The effect of spatial frequency on global precedence and hemispheric differences. Perception \& Psychophysics, 59, 187-201.

HÜBNER, R. (1998). Hemispheric differences in global/local processing revealed by same-different judgements. Visual Cognition, 5, 457-478.
HÜBNER, R., \& MALINOWSKI, P. (2002). The effect of response competition on functional hemispheric asymmetries for global/local processing. Perception \& Psychophysics, 64, 1290-1300.

HÜBNER, R., \& VolBERG, G. (2005). The integration of object levels and their content: A theory of global/local processing and related hemispheric differences. Journal of Experimental Psychology: Human Perception \& Performance, 31, 520-541.

IVRY, R. B., \& RoberTSON, L. C. (1998). The two sides of perception. Cambridge, MA: MIT Press.

LAMB, M. R., \& RoberTson, L. C. (1989). Do response time advantage and interference reflect the order of processing of global- and locallevel information? Perception \& Psychophysics, 46, 254-258.

LAMB, M. R., RoberTson, L. C., \& KNIGHT, R. T. (1989). Attention and interference in the processing of global and local information: Effects of unilateral temporal-parietal junction lesions. Neuropsychologia, 27, 471-483.

Lux, S., Marshall, J. C., Ritzl, A., Weiss, P. H., Pietrzyk, U., Shah, N. J., ET AL. (2004). A functional magnetic resonance imaging study of local/global processing with stimulus presentation in the peripheral visual hemifields. Neuroscience, 124, 113-120.

Malinowski, P., HÜBner, R., KeIL, A., \& Gruber, T. (2002). The influence of response competition on cerebral asymmetries for processing hierarchical stimuli revealed by ERP recordings. Experimental Brain Research, 144, 136-139.

MarTin, M. (1979). Hemispheric specialization for local and global processing. Neuropsychologia, 17, 33-40.

PAQUeT, L. (1992). Global and local processing in nonattended objects: A failure to induce local processing dominance. Journal of Experimental Psychology: Human Perception \& Performance, 18, 512-529.

Paquet, L., \& Merikle, P. M. (1988). Global precedence in attended and nonattended objects. Journal of Experimental Psychology: Human Perception \& Performance, 14, 89-100.

Polster, M. R., \& Rapcsak, S. Z. (1994). Hierarchical stimuli and hemispheric specialization: Two case studies. Cortex, 30, 487-497.

Robertson, L. C., \& Delis, D. C. (1986). "Part-whole" processing in unilateral brain-damaged patients: Dysfunction of hierarchical organization. Neuropsychologia, 24, 363-370.

Sasaki, Y., Hadithhani, N., Fischl, B., Liu, A. K., Marrett, S., Dale, A. M., \& Tootell, R. B. (2001). Local and global attention are mapped retinotopically in human occipital cortex. Proceedings of the National Academy of Sciences, 98, 2077-2082.

Schatz, J., Craft, S., Koby, M., \& DeBaun, M. R. (2004). Asymmetries in visual-spatial processing following childhood stroke. Neuropsychology, 18, 340-352.

VAN KLEECK, M. H. (1989). Hemispheric differences in global versus local processing of hierarchical visual stimuli by normal subjects: New data and a meta-analysis of previous studies. Neuropsychologia, 27, 1165-1178

Volberg, G., \& HüBner, R. (2004). On the role of response conflicts and stimulus position for hemispheric differences in global/local processing: An ERP study. Neuropsychologia, 42, 1805-1813.

VOLBERG, G., \& HÜBNER, R. (2006). Hemispheric differences for the integration of stimulus levels and their contents: Evidence from bilateral presentations. Perception \& Psychophysics, 68, 1274-1285.

VOLBERG, G., \& HüBNER, R. (2007). Deconfounding the effects of congruency and task difficulty on hemispheric differences in global/local processing. Experimental Psychology, 54, 83-88.

Yovel, G., Levy, J., \& Yovel, I. (2001). Hemispheric asymmetries for global and local visual perception: Effects of stimulus and task factors. Journal of Experimental Psychology: Human Perception \& Performance, 27, 1369-1385.

(Manuscript received December 2, 2005; revision accepted for publication August 2, 2006.) 